\title{
An Efficient Feature Specific Neural Network for Multi Class Classification
}

\author{
T.Ravichandran, Krishna Mohanta, C.Nalini
}

\begin{abstract}
There has been global resurgence of interest in herbal drugs in the recent past. Though herbal medicines are effective in the treatment of various ailments very often these drugs are unscientifically exploited or improperly used. Therefore, these herbal drugs deserve detailed studies in the light of modern medicine. In spite of synthetic drugs, herbal drugs have their place in therapy. Their effectiveness, low-cost and comparative freedom from serious toxic effects makes these medicines not only popular but also an acceptable mode of treating diseases even in modern times. Medicinal plants are those plants that are used in treating and preventing specific and human has been using herbs for generations around the world, due to charm needed to cure the disease, many people have come to the conclusion that even chemical drugs their answers may already be sick of these medications may be harmful for health them in the future. Still, the use of plants as a source of medicine is very much important for human beings. Identify medicinal and how to use them is so important.
\end{abstract}

Keywords: Degeneration, Drugs, macromolecules, toxicity.

\section{INTRODUCTION}

We propose another training technique, in particular Sampling, to upgrade the proficiency of the learning procedure of FENN. learning structure is a basic usage of the rule of divided redundancy for learning [12], which incorporates expanding interims of time between resulting surveys of already learnt material so as to abuse the mental separating impact. In spite of the fact that the guideline of dispersed redundancy is valuable in numerous unique circumstances, it requires a student to obtain an extensive number of things and hold them in memory inconclusively. he Sampling preparing technique proposed in this paper gets a near plan to structure a learning count for FENN. To this completion, each preparation test is designated to a carton with a quantity work that is capably adjusted by the grouping certainty given by the FENN softmax yield. After a learning accentuation, tests with raised certainty will be set for a situation with low appearance probability, while those with low certainty will be set for another situation with high appearance probability, along these lines they will undoubtedly appear for assurance as the preparation information in the accompanying cycle.

Starting late, neural system troupe techniques have expanded broad energy among researchers in AI arrange. Among

Revised Manuscript Received on July 10, 2019.

T.Ravichandran, Research Scholar, Bharath Institute of Higher Education and Research. Chennai -600073, Tamilnadu, India.

Dr.Krishna Mohanta, Associate Professor, Department of CSE,Kakatiya Institute of Technology and Science, Telangana, India.

Dr.C.Nalini, Professor, Department of CSE, Bharath Institute of Higher Education and Research, Chennai, Tamil Nadu, India. various varieties, the most outstanding consolidate packing [16], arcing [8] or boosting [9]. The essential idea of these techniques is to create various variations of a pointer. Exactly when expectations from these interpretations are joined, logically enduring and spread forecasts are produced. At the point while associated with systems, these frameworks can yield enthusiastic redesigns in theory execution [10]. The reason behind this is because neural systems are normally touchy [13], for instance little changes in preparing set just asparameter assurance may convey extensive changes inexecution. Most of blend procedures for classifiers acknowledge that the classifiers molding the classifier troupe must be both contrasting and exact. The "varying assortment" doubt suggests that the classifiers need to make free order mistakes, to improve overall expectation exactness. Both theoretical [17] and observational work $[9,10]$ has exhibited that a nice neural system troupe is one where the individual systems are both precise and make mistakes on different bits of the data space. For example, In [7] have exhibited that a various classifier system reliant on an essential bigger part mix standard can give commonly magnificent upgrades in precision at whatever point solidified classifiers make free blunders.

The remainder of the paper is arranged as pursues. Segment 2 clarifies related examinations detailed in the writing. Segment 3 present the proposed FENN auxiliary plan and the designs utilized. Area 4 introduces the trial results and investigation. In conclusion, Section 5 abridges our outcome and finishes up the paper

\section{REALTED WORKS}

For a serious in length time, neural systems have shown their ability in AI. To assemble arrange execution, a couple of examiners rely upon increasingly significant systems [18] while others propose including interstate information [19]. A champion among the most testing pieces of significant systems is the methods by which to plan them and sweep for their hyper parameter regards. To address this issue, some proposed strategies fuse the usage of stochastic significance [20] or thick convolution systems [21]. Nevertheless, the limit of these systems is that all proposed significant system 
structures are deterministic which limits the flexibility of the models and accordingly rouses us to design an automated search for perfect CNN hyper parameters In [3] and [4], proposed strategies rely upon Bayesian improvement methodology and yield better execution. In this paper we look into a heuristic chase. Our technique relies upon hereditary figuring most likely comprehended for non-deterministic issue objectives. Our work means to plan and assessment a contender new GA encoding system for CNN structure look for. Throughout late decades, different assessments have investigated HCCR [16], realizing the headway of various procedures, for instance, non-direct standardization, information increment, include extraction, and quadratic classifier modification.

\section{FEATURE BASED ENSAMPLES NEURAL NETWORKS (FENN)}

\subsection{Pooling}

The general Neural network is appeared in Figure 1. There are three concealed layers with nonlinear units, and four layers of weights. The principal layer of weights is the convolutional filter layer. The second layer is a pooling (low pass filtering) and downsampling layer. The third layer is an arbitrary projection layer. The fourth layer is the main prepared layer. The yield layer has linear units. The network can be adroitly separated into two phases, that as far as anyone is concerned have not recently been consolidated. The primary stage is the convolution tuple extraction organize, and to a great extent pursues that of existing ways to deal with image classification [21]. The second stage is the classifier arrange, and to a great extent pursues the methodology of $[11,14]$. We currently depict the two phases in detail

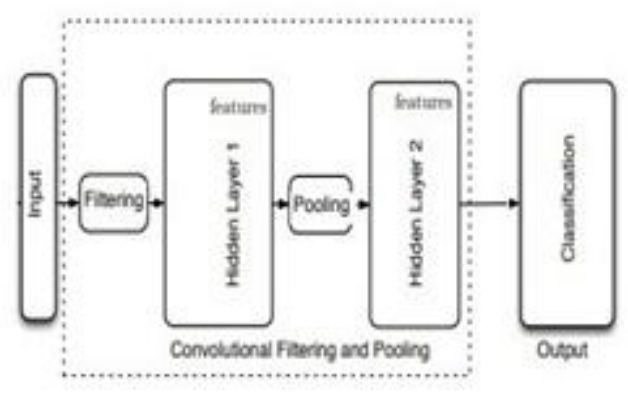

Figure 1. Convolution Filter and Pooloing

The functions are nonlinear changes connected termwise to framework contributions to deliver network yields of a similar size. In any case, the accompanying scientific definition of the calculation demonstrates a standard layered neural network plan of this calculation is pertinent, as appeared in Figure 1, and along these lines that calculation of all features can be acquired in one shot from a K-section grid containing a group of $\mathrm{K}$ training focuses. The way to this detailing is to take note of that since convolution is a linear administrator, a grid can be built that when increased by a data network creates a similar outcome as Convolution feature identification. Convolution connected to one example of the data

\subsection{Training method}

Propelled by this idea, we propose another FENN training answer for location the three issues referenced previously. The proposed training technique is called FallSample; it sorts the training dataset into gatherings and utilizations the softmax yield to progressively and consequently change the chose quotas of samples in various gatherings. The yield layer with k-route softmax as the actuation work generates a likelihood dissemination over the $\mathrm{n}$ classes. As per a past report, the softmax yield of $\mathrm{CNN}$ can be viewed as a confidence estimation of the CNN categorization yield [40]. On account of training utilizing FallSample, samples with elevated confidence are set in a container with low look likelihood and those with low confidence are disposed of so the rest of the samples can be much of the time inspected in the network.

\section{Algorithm 1: Training}

Input: training set

Output: network parameters

Initialization: learning rate; quota; quota-updating

1: while not converge do

2: sample a batch from $\mathrm{X}$

3: get the softmax output of the predicted class from forward propagation:

4: calculate error term using back propagation:

5: update network parameters

6: calculate quota-updating function

7: update quota parameters

8: end while

\subsection{Neural Network Ensembles}

In order to show signs of improvement the overall rightness, an outfit of classifiers must be both exact notwithstanding various. To make an outfit of classifiers in this examination, two philosophies are used. The first relies upon stowing, where a well known real re-inspecting system called bootstrap [17] is used to produce different preparing sets on which the individual systems from a group are created. Nevertheless, the different assortment of individual classifiers were tuned not simply by moving examples of the preparation set, yet what's more through different early on weight settings, learning calculations, number of concealed neurons and number of preparing ages. A subsequent

Blue Eyes Intelligence Engineering

\& Sciences Publication 
approach used standard AdaBoost.M2 system [3], since the additional randomization procedures used in stowing did notbasically improve the gathering execution. In each cycle, a delicate learning estimation is called and gave a substitute dispersal Dt that is changed by underlining explicit preparing points of reference. The flow is invigorated to give wrong characterizations higher loads than right groupings. The entire weighted preparing set is given to the fragile understudy to process the weak theory ht. A classifier impact $\beta$ is enrolled, which is used in the last weighted vote. Close to the end, each and every frail theory are joined into a single speculation.

\section{RESULTS:}

Data gathering and preprocessing are the underlying phases of the data mining process. Since just legitimate data will deliver accurate yield, data preprocessing is the key stage. For this examination, we utilize the Weather data from UCI. We simply think about related data and disregard the rest. These models are the equivalent in both the training test and the predicted test in the examination test. For the evaluation of the algorithms' outcomes, alignment, cross-approval and outer testing were done. The staying $20 \%$ of the data were utilized as the test set to approve the model accuracy.

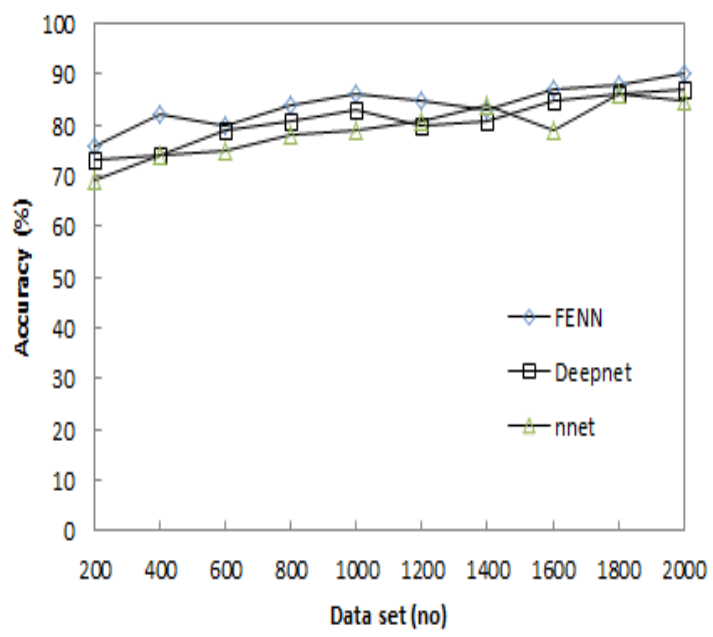

Fig.2 Accuracy

It very well may be seen from the Figure 2 that, since the model depends on this data set, the accuracy rate is very high, and the accuracy of certain data is near $100 \%$. Be that as it may, with the expansion of data measure, the accuracy rate has declined and has turned out to be shaky. The nnet model gets the best classification accuracy when the quantity of samples is 800 , however then it has poor execution. So also error rate are appeared in fig 3 . Which is obviously demonstrates that FENN give the most reduced error rate when contrast with the deepnet and nnet

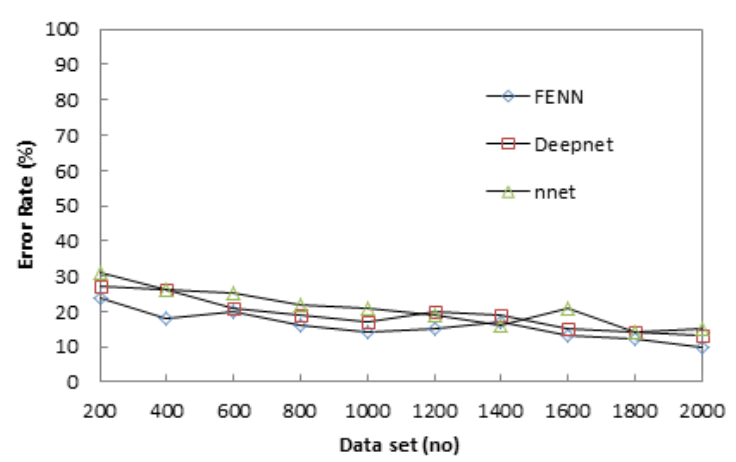

Fig.3 Error Rate

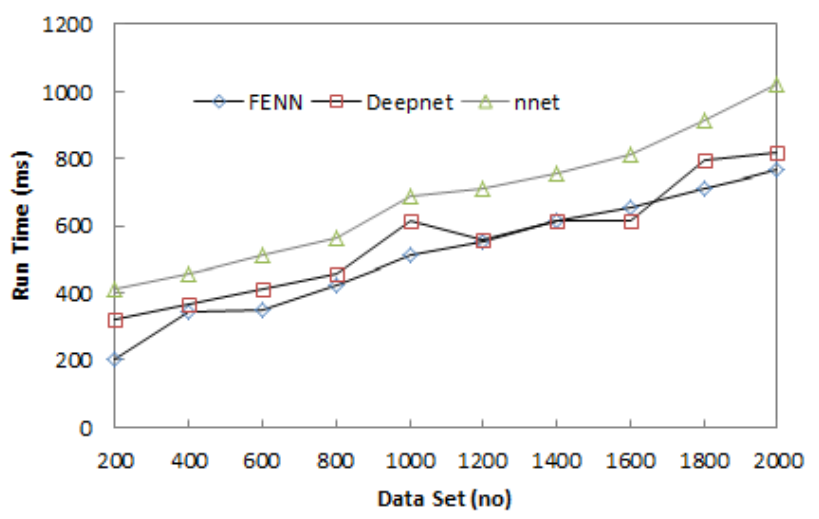

Fig.4 Runtime

The curve of nnet is on the ascent, and the pre-development rate is the fastest among the four strategies. With the expansion in the quantity of samples, the time required to set up the model turns out to be amazingly unsteady. In general, the FENN strategy sets aside the littlest effort to figure.

\section{CONCLUSION}

This paper additionally proposed another training technique for FENN, through the effective utilization of training samples. A FENN that is prepared with the example method centers around befuddling samples and specifically disregards well-perceived samples, while adequately staying away from obstruction because of uproarious samples. Trial results on a few data sets show that the proposed strategies for pruning classifiers can viably accomplish comparative better prediction accuracy.

\section{REFERENCES}

1. Karpathy \& Fei-Fei,, "Deep visual-semantic alignments for generating image descriptions", In Proceedings of the IEEE Conference on Computer Vision and Pattern Recognition., pp. 3128-3137, 2016.

2. Garcia D, Parès F \& Suzumura. "On the Behavior of Convolutional Nets for Feature Extraction", Journal of Artificial Intelligence Research, Vol.61, pp. 563-592, 2018 
3. Y. Taigman, M. Yang, M. Ranzato and L. Wolf. Deepface: Closing the gap to human-level performance in face verification. In Computer Vision and Pattern Recognition (CVPR), pp. 1701-1708, 2014

4. Ham \& Kostanic, "Principles of Neurocomputing for Science and Engineering", McGraw-Hill Higher Education, 2000.

5. LeCun, Bengio \& Hinton, " Deep Learning", Nature, vol.521, no.7553, pp. 436-444, 2015

6. Jarrett, Kavukcuoglu, Ranzato \& LeCun, "What is the best multi-stage architecture for object recognition?",In Proceedings of the IEEE International Conference on Computer Vision", pp. 2146- 2153, 2009.

7. Nair \& Hinton, "Rectified linear units improve restricted boltzmann machines", In Proceedings of International Conference on Machine Learning, pp. 807-814, 2010. 